\title{
Quantum machine intelligence
}

\section{Launching the first journal in the area of quantum artificial intelligence}

\author{
Giovanni Acampora ${ }^{1}$ \\ Published online: 23 May 2019 \\ (C) Springer Nature Switzerland AG 2019
}

Quantum computing is a fascinating research area at the intersection of computer science, physics, and engineering, which is catching the attention of both the academic and corporate worlds by promising a revolution in computing performance, due to a massive and intrinsic parallelism enabled by "interfering, super-positioning, and entangling" different pieces of information. Although it was initially thought of as a way to efficiently simulate quantum mechanics on a computer, today, research on quantum computing is focusing on the so-called quantum advantage or quantum supremacy - the design of quantum algorithms offering significant speedup compared to the best possible algorithm on a classical computer-to spur the development of new breakthroughs in different application domains, such as chemistry, medicine, and financial services, just to name a few. This research is particularly significant because the quantum computation is no longer a theoretical utopia since, currently, real quantum computers can be accessed and programmed through an Internet connection, and everyone can try their hand at implementing wellestablished quantum algorithms, such as Shor's and Grover's algorithms (Shor 1994; Grover 1996), or designing completely new quantum algorithms.

In this pioneering scenario, the recent implementation of quantum algorithms for machine learning has led to a flurry of increasingly sophisticated results that show how quantum computers could be efficient in solving problems in the field of artificial intelligence faster than their classical counterparts (Biamonte et al. 2017; Schuld et al. 2014; Dunjko and Briegel 2018; Schuld and Petruccione 2018). The big advantage of quantum computing applied

Giovanni Acampora

giovanni.acampora@unina.it

1 Department of Physics "Ettore Pancini”, University of Naples Federico II, Naples, Italy to machine learning is due to the exponential increase in the number of dimensions it can process with respect to classical machine learning algorithms (Havlícek et al. 2019). As an example, while a classical artificial neuron can process an input of $N$ dimensions, a quantum perceptron can process $2^{N}$ dimensions, and it can tremendously speed up the running time of both training and classification algorithms (Tacchino et al. 2019). Moreover, from a different point of view, particularly exciting is the prospect of using classical algorithms of machine learning for the discovery and design of quantum materials, devices, algorithms, and circuits. In this context, the current skills and expertise gained in classical machine learning will enable a rapid growth in developing new approaches for the automatic design of "quantum things." As a consequence, the scientific community agrees that artificial intelligence could be a killer application for future generations of computational devices based on quantum technologies. The strong interest in the integration of quantum computing and artificial intelligence is further proved by the growing number of conferences, workshops, and social network activities that, from 2014, take place every year on this topic. As an example, Fig. 1 shows the rapid growth in the number of papers published in the area of quantum machine intelligence from 2012 to present. ${ }^{1}$

However, in spite of the strong interest shown by the scientific community for the synergistic area of quantum computing and artificial intelligence, this research field lacks a scientific journal devoted to publishing the main research results in this area. For this reason, it is my pleasure to introduce Quantum Machine Intelligence, a new Springer journal representing the first scientific forum where researchers and practitioners will promptly share the latest developments and research results about the revolutionary world of integration between quantum computing and

\footnotetext{
${ }^{1}$ Scopus search query: ( TITLE-ABS-KEY ( "quantum" AND "artificial intelligence") OR TITLE-ABS-KEY ( "quantum" AND "machine learning") OR TITLE-ABS-KEY ( "quantum" AND "machine intelligence" ) ) AND PUBYEAR > 2011
} 


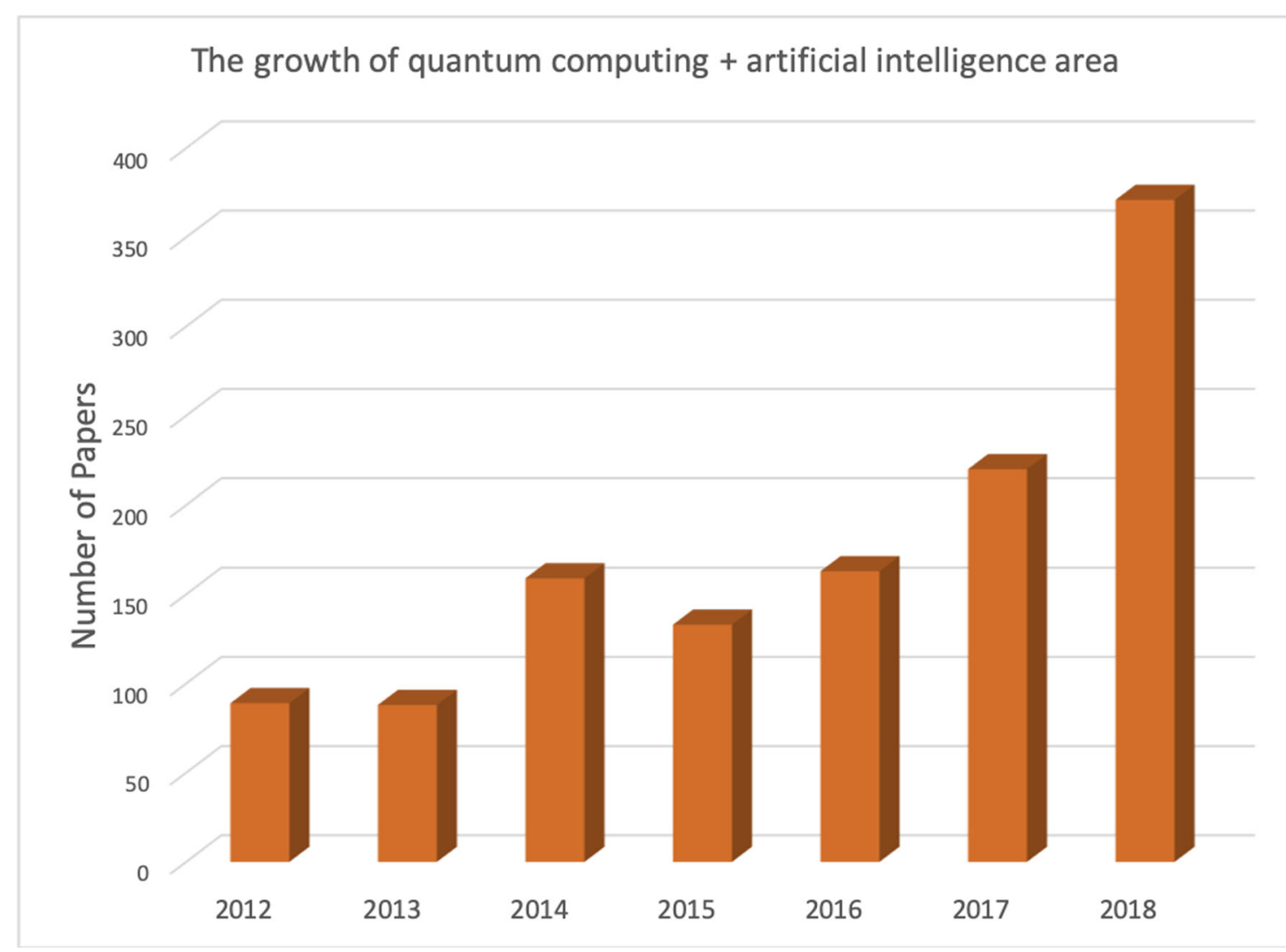

Fig. 1 The growth of the numbers of papers published in the area of quantum machine intelligence from 2012 to present (source: Scopus)

artificial intelligence. Quantum Machine Intelligence will represent the main reference for this pioneering research field by promoting a synthesis of machine learning, data science, and computational intelligence research with quantum computing developments. The activity of the journal will be carried on by a set of Field Editors and an Editorial Board composed of top scientists in the fields of computer science, physics, and engineering. The Field Editors are the persons in charge of supervising the various research sections of the journal.

The journal is organized into five different research sections to cover all the possible aspects of the synergy between quantum computing and artificial intelligence. The section named Quantum Machine Learning, supervised by Seth Lloyd, Massachusetts Institute of Technology (USA), publishes research papers dealing with the quantum implementation of the machine learning algorithms such as quantum perceptrons, quantum neural networks, and quantum clustering. The second section, Quantum Computing for Artificial Intelligence, supervised by Hans J. Briegel from University of Innsbruck (Austria), is aimed at publishing papers related to quantum-enhanced methods for artificial intelligence, including models for quantum artificial agents, quantum reinforcement learning, quantum reasoning, and the use of machine learning in quantum experiments. The third section, Artificial Intelligence for Quantum Information Processing, supervised by Chin-Teng Lin, University of Technology Sydney (Australia), is devoted to publishing papers related to the exploitation of classical artificial intelligence algorithms to solve hard quantum problems such as the automatic design of quantum circuits, and quantum algorithm discovering. The fourth section, Quantumand Bio-inspired Computational Intelligence, supervised by Francisco Herrera, University of Granada (Spain), publishes manuscripts where new artificial intelligence methodology, inspired by quantum effects or biological phenomena, is introduced to improve the performance of current artificial intelligence approaches; some examples of such research activities are quantum-inspired evolutionary strategies. The last section, Quantum Optimization, supervised by Davide Venturelli, Universities Space Research Association (USA), is aimed at publishing research manuscripts concerning the design of quantum and quantum-inspired algorithms, including variational, hybrid, and based on the 
quantum annealing paradigm, for solving hard optimization problems.

In this organizational scenario, my role as the Editorin-Chief will be aimed at coordinating all the editorial activities to ensure appropriate-level review of the articles in terms of scientific quality and reviewing time, and attracting high-class papers by strongly involving quantum computing and artificial intelligence communities in all the journal activities.

The launch of a new journal is comparable to the birth of a child: its delivery may appear simple and natural, but its growth depends on the quality of the attention it receives from the people around it. In this mission, the editorial team is committed to supporting all publishing activities by undertaking all the necessary steps to make Quantum Machine Intelligence a top-leading journal in its area of competence. At the same time, we rely on the entire scientific community to publish relevant and remarkable contributions in the field of quantum computing and artificial intelligence.

Finally, we are honored to introduce to you the first issue of the journal, which would not have been possible to print without the valuable support from the Field Editors, Editorial Board members, and reviewers, and from Springer. Hoping the publication of this issue will stimulate and start new research activities into the pioneering area of quantum computing and artificial intelligence, we wish you a pleasant reading of the first papers published in Quantum Machine Intelligence.
With enthusiasm,

Giovanni Acampora

Editor-in-Chief

University of Naples Federico II, Italy

\section{References}

Biamonte J, Wittek P, Pancotti N, Rebentrost P, Wiebe N, Lloyd S (2017) Quantum machine learning. Nature 549:195-202

Dunjko V, Briegel HJ (2018) Machine learning \& artificial intelligence in the quantum domain: a review of recent progress. Rep Prog Phys 81(7):074001

Grover LK (1996) A fast quantum mechanical algorithm for database search. In: Proceedings of the 28th annual ACM symposium on theory of computing, STOC'96. ACM, New York, pp 212-219

Havlícek V, Córcoles AD, Temme K, Harrow AW, Kandala A, Chow JM, Gambetta JM (2019) Supervised learning with quantumenhanced feature spaces. Nature 567(7747):209-212

Schuld M, Petruccione F (2018) Supervised learning with quantum computers. Quantum science and technology. Springer International Publishing

Schuld M, Sinayskiy I, Petruccione F (2014) An introduction to quantum machine learning. Contemp Phys 56:09

Shor PW (1994) Algorithms for quantum computation: discrete logarithms and factoring. In: Proceedings 35th annual symposium on foundations of computer science, pp 124-134

Tacchino F, Macchiavello C, Gerace D, Bajoni D (2019) An artificial neuron implemented on an actual quantum processor. npj Quantum Inf 5(1):26

Publisher's note Springer Nature remains neutral with regard to jurisdictional claims in published maps and institutional affiliations. 